\title{
Contractually stable networks
}

J.F. Caulier, A. Mauleon and V. Vannetelbosch

Discussion Paper 2008-45

Département des Sciences Économiques

de l'Université catholique de Louvain 


\title{
CORE DISCUSSION PAPER
}

2008/66

\section{Contractually stable networks}

\author{
Jean-François CAULIER ${ }^{1}$, Ana MAULEON ${ }^{2}$ \\ and Vincent VANNETELBOSCH ${ }^{3}$
}

December 2008

\begin{abstract}
We develop a theoretical framework that allows us to study which bilateral links and coalition structures are going to emerge at equilibrium. We define the notion of coalitional network to represent a network and a coalition structure, where the network specifies the nature of the relationship each individual has with his coalition members and with individuals outside his coalition. To predict the coalitional networks that are going to emerge at equilibrium we propose the concept of contractual stability which requires that any change made to the coalitional network needs the consent of both the deviating players and their original coalition partners. We show that there always exists a contractually stable coalitional network under the simple majority decision rule and the component-wise egalitarian or majoritarian allocation rules. Moreover, requiring the consent of group members may help to reconcile stability and efficiency.
\end{abstract}

Keywords: networks, coalition structures, contractual stability, allocation rules.

JEL Classification: A14, C70

\footnotetext{
${ }^{1}$ Facultés Universitaires Saint-Louis, CEREC, B-1000 Bruxelles, Belgium.

${ }^{2}$ Facultés Universitaires Saint-Louis, FNRS \& CEREC, B-1000 Bruxelles, Belgium; Université catholique de Louvain, CORE, B-1348 Louvain-la-Neuve, Belgium.

${ }^{3}$ Université catholique de Louvain, FNRS \& CORE, B-1348 Louvain-la-Neuve, Belgium. E-mail:

Vincent.vannetelbosch@uclouvain.be. This author is also member of ECORE, the newly created association between CORE and ECARES

This paper was reviewed in NAJ: Not a Journal 13.

This paper presents research results of the Belgian Program on Interuniversity Poles of Attraction initiated by the Belgian State, Prime Minister's Office, Science Policy Programming. The scientific responsibility is assumed by the authors.
} 



\section{Introduction}

The organization of individual agents into networks and groups has an important role in the determination of the outcome of many social and economic interactions. For instance, networks of personal contacts are important in obtaining information about job opportunities. Goods can be traded and exchanged through networks, rather than markets, of buyers and sellers. Networks also play important roles in providing mutual insurance especially in developing countries. ${ }^{1}$ Partitioning of societies into groups is also important in many contexts, such as the provision of public goods and formation of alliances, cartels and federations. The understanding of how and why such networks and groups form and the precise way in which they affect outcomes of social and economic interactions has been apprehended separately by the coalition theory and the network theory.

One limit of both theories is that it cannot incorporate the existence of bilateral agreements among agents belonging to different coalitions - that is commonly observed in many situations. A first situation has to do with the formation of $R \& D$ joint ventures and of bilateral R\&D collaborations. On the one hand, Bloch (1995) has analyzed the formation of associations of firms, like $R \& D$ joint ventures or groups of firms adopting common standards, in an oligopolistic industry. On the other hand, Goyal and Moraga-González (2001) or Goyal and Joshi (2003) have analyzed the incentives for R\&D collaboration between horizontally related firms by considering that collaboration links are bilateral and are embedded within a broader network of similar links with other firms. However, it may happen that firms A and B may decide to form an R\&D joint venture while firms B and $\mathrm{C}$ sign a bilateral R\&D agreement. What is the architecture of the resulting collaboration network and the structure of associations that are likely to emerge?

A second situation has to do with the formation of free-trade agreements and customs unions. On the one hand, Yi (1996) has studied the incentives for countries to form regional free trade associations and customs unions, and the strategic stability of those particular trading regimes. On the other hand, Goyal and Joshi (2005) and Furusawa and Konishi (2006) have investigated the formation of bilateral free trade agreements as a network formation game. Thus, the literature has considered that countries participate either in the formation of bilateral free trade agreements or in the formation of customs unions or multilateral free trade agreements, but not in the formation of both types of agreements at the same time. This is a strong restriction indeed since many countries are involved in both types of agreements at the same time: Mexico belongs to NAFTA and has a bilateral free trade agreement with the European Community, while the United States have no specific trade agreement with the European Community. What are the

\footnotetext{
${ }^{1}$ Jackson $(2003,2005)$ provides surveys of models of network formation.
} 
incentives for countries to form bilateral free trade agreements and/or customs unions?

There are many other situations where agents are part of a network and belong to groups or coalitions. It is very common that firms having some common interest within an industry regroup themselves into an industry association. Moreover, in various industries, such as automobiles, clothing, electronics, pharmaceuticals, and food, manufacturers develop networks of exchange both with input suppliers and retailers or wholesalers. ${ }^{2}$ In labour markets, workers are linked to each other within each firm through a hierarchy that is, a network - and, at the same time workers may group themselves into unions. Municipalities are connected to each other through a network of roads, railway tracks or waterways, and they may share some common facilities or emergency services. Individuals are living their social interactions in clubs or communities as well as through friendship networks.

The aim of this paper is to develop a theoretical framework that allows us to study which bilateral links and coalition structures are going to emerge at equilibrium. We define the notion of coalitional network to represent a network and a coalition structure, where the network specifies the nature of the relationship each individual has with his coalition members and with individuals outside his coalition. This new framework forces us to redefine key notions of theory of networks, value and allocation rules, and to introduce a new solution concept : contractual stability.

The idea of contractual stability is that adding or deleting a link needs the consent of coalition partners. For instance, in the context of R\&D alliances, firms may decide to have a common laboratory with some partners, while developing bilateral R\&D agreements with other partners. The signing of a bilateral R\&D agreement may need the consent of those partners within the common laboratory or joint venture. Moreover, the formation of new coalition structures may need the consent of original coalition partners. Thus, once a coalition has been formed, the consent of coalitional partners is required in order to add or delete links that affect some coalition partners, or to modify the existing coalition. As in Drèze and Greenberg (1980) the word "contractual" is used to reflect the notion that coalitions are contracts binding all members and subject to revision only with consent of coalitional partners. ${ }^{3}$ Two different decision rules for consent are analyzed:

\footnotetext{
${ }^{2}$ Kranton and Minehart (2000) have analyzed the endogenous formation of networks between input suppliers and manufacturers while Mauleon, Sempere and Vannetelbosch (2005) have studied the formation of networks between manufacturers and retailers. Wang and Watts (2006) have examined the formation of buyer-seller networks when sellers can form an association of sellers to pool their customers.

${ }^{3}$ One example mentioned by Drèze and Greenberg (1980) are rules governing entry and exit in labor cooperatives. A new partner will enter the cooperative only if (i) he wishes to come in; (ii) his new partners wish to accept him; and (iii) he obtains from his former partners permission to withdraw (only if he was before member of another cooperative).
} 
simple majority or unanimity. ${ }^{4}$ When allocations do not depend on coalition structures, there always exists a contractually stable coalitional network under the unanimity decision rule. Looking at some classical models from network theory (co-author model, symmetric connections model, model of buyer-seller networks), we observe that requiring the consent of group members under the simple majority may help to reconcile stability and efficiency. When allocations depend on coalition structures, there always exists a contractually stable coalitional network under the simple majority decision rule and the component-wise egalitarian or majoritarian allocation rules. However, if the component-wise dictatorial allocation rule is adopted, then a contractually stable coalitional network always exists only under the unanimity decision rule.

The paper is organized as follows. In Section 2 we introduce the framework of coalitional networks. In Section 3 we define the concept of contractual stability. In Section 4 we derive some results about the existence of contractually stable networks and we look whether efficient coalitional networks are likely to be stable or not. In Section 5 we comment upon some of the features of the framework showing that it is general enough to study the emergence of community structures. In addition, it can be extended so that overlapping collections of individuals may arise. In Section 6 we conclude.

\section{Coalitional networks}

Let $N=\{1, \ldots, n\}$ be the finite set of players who are connected in some network relationship and who belong to some coalitions or communities. A coalitional network $(g, P)$ consists of a network $g \in G^{N}$ and a coalition structure $P \in \mathbb{P}$. A network $g$ is simply a list of which pairs of players are linked to each other with $i j \in g$ indicating that $i$ and $j$ are linked under the network $g$. Let $G^{N}=\left\{g: g \subset g^{N}\right\}$ denote the set of all possible networks on $N$ and $g^{N}$ is the set of all subsets of $N$ of size 2. A coalition structure $P=\left\{S_{1}, S_{2}, \ldots, S_{m}\right\}$ is simply a partition of the player set $N, S_{a} \cap S_{b}=\varnothing$ for $a \neq b$, $\cup_{a=1}^{m} S_{a}=N$ and $S_{a} \neq \varnothing$ for $a=1, \ldots, m$. Let $\# S_{a}$ be the cardinality of coalition $S_{a}$ and $S(i) \in P$ be the coalition whose player $i$ belongs. Let $\mathbb{P}$ denote the finite set of coalition structures. A sub-coalitional network of $(g, P)$ is $(h, Q)$ with $h \subset g$ and $Q \subset P$. For instance, if $N=\{1,2,3,4,5,6,7,8\}$, then $(g, P)=(\{12,23,45,56,78\},\{\{1\},\{2,3,4,5\}$, $\{6,7,8\}\})$ is the coalitional network in which there is a link between players 1 and 2 , a link between players 2 and 3, a link between players 4 and 5 , a link between players 5 and 6 , and a link between players 7 and 8 , and players $2,3,4$ and 5 are in the same coalition while players 6,7 and 8 are in another coalition and player 1 is alone. This coalitional

\footnotetext{
${ }^{4}$ For instance, the unanimity decision rule is used in the European Community whenever a new bilateral free trade agreement is proposed by any country member.
} 
network $(g, P)=(\{12,23,45,56,78\},\{\{1\},\{2,3,4,5\},\{6,7,8\}\})$ is depicted in Figure 1.

Figure 1: A coalitional network

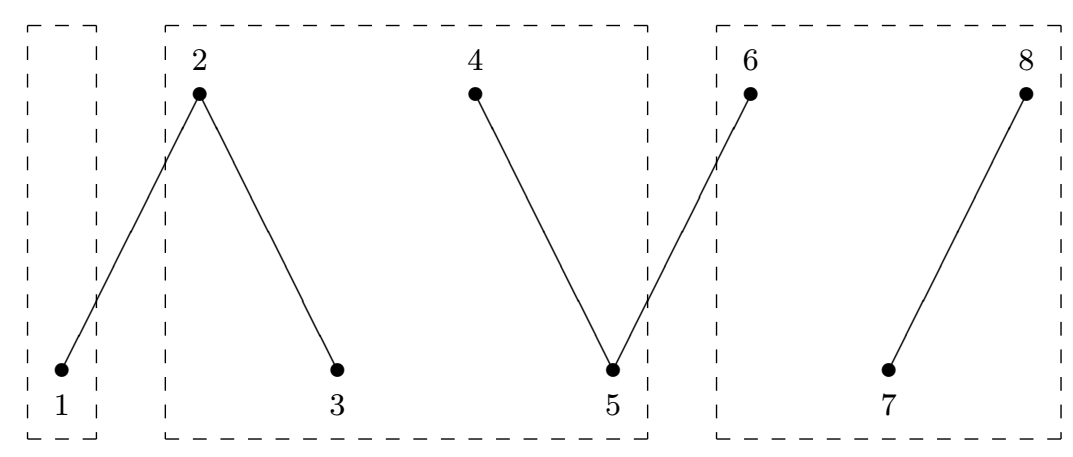

Let $N(g)=\{i \mid \exists j$ such that $i j \in g\}$ be the set of players who have at least one link in the network $g$. Let $n(g)$ be the cardinality of $N(g)$. For any $Q \subset P, N(Q)=\{i \in S: S \in$ $Q\}$ is the set of players that belong to some coalition $S \in Q$. Let $N(g+Q)=N(g) \cup N(Q)$. Finally, let $N(g, P)$ be the set of players who have at least one link in the network $g$ or that belong to a coalition $S \in P$ such that at least one member of $S$ has a link in the network $g$.

Definition 1 A component of a coalitional network $(g, P)$ is a nonempty sub-coalitional network $(h, Q)$, with $h \subset g$ and $Q=\left\{S_{k}: S_{k} \in P\right\} \subset P$, such that

(i) $h=\left\{i j \in g: \exists S, S^{\prime} \in Q\right.$ such that $i \in S$ and $\left.j \in S^{\prime}\right\}$,

(ii) for all $S, S^{\prime} \in Q$ there exists a sequence of coalitions $S^{1}, S^{2}, \ldots, S^{K}$ with $S^{1}=S$ and $S^{K}=S^{\prime}$ such that for any $l \in\{1, \ldots, K-1\}, S^{l} \in Q$ and there exists $i_{l} i_{l+1} \in h$ with $i_{l} \in S^{l}$ and $i_{l+1} \in S^{l+1}$.

A component $(h, Q)$ of $(g, P)$ consists of a nonempty sub-network $h$ of $g$ and the coalitions in $P$ that contain at least one player with a link in $h$. The set of components of $(g, P)$ is denoted as $C(g, P)$. Under this definition of a component, a coalition whose members have no links is not considered as a component.

Definition 2 A sub-coalitional network $(h, Q)$ is connected if for each $i \in N(h+Q)$ and $j \in N(h+Q)$ there exists a sequence of coalitions $S^{1}, S^{2}, \ldots, S^{K}$ with $i \in S^{1} \in Q$ and $j \in S^{K} \in Q$ such that for any $l \in\{1, \ldots, K-1\}, S^{l} \in Q$ and there exists $i_{l} i_{l+1} \in h$ with $i_{l} \in S^{l}$ and $i_{l+1} \in S^{l+1}$.

Consider the coalitional network $(\{12,23,45,56,78\},\{\{1\},\{2,3\},\{4,5\},\{6,7,8\}\})$. The connected sub-coalitional networks are $(\{12,23\},\{\{1\},\{2,3\}\}),(\{23\},\{\{2,3\}\}),(\{12\}$, 
$\{\{1\},\{2,3\}\}),(\{45,56,78\},\{\{4,5\},\{6,7,8\}\}),(\{45\},\{\{4,5\}\}),(\{56\},\{\{4,5\},\{6,7,8\}\})$, $(\{78\},\{\{6,7,8\}\})$. The components are the maximal connected sub-coalitional networks, that is $(\{12,23\},\{\{1\},\{2,3\}\})$ and $(\{45,56,78\},\{\{4,5\},\{6,7,8\}\})$. These two components are depicted in Figure 2.

Figure 2: A coalitional network and its components

\begin{tabular}{|c|c|c|c|}
\hline$\ulcorner-\neg$ & $\ulcorner-----\neg$ & $\ulcorner-----\neg$ & $\ulcorner---------\neg$ \\
\hline 1 & 2 & 4 & $\begin{array}{ll}1 & 6\end{array}$ \\
\hline & 8 & I & \\
\hline & 1 & & 1 \\
\hline 1 & 1 & 1 & 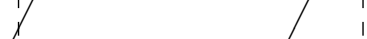 \\
\hline & । & 1 & 1 \\
\hline 1 & I & 1 & 1 \\
\hline 1 & 1 & 1 & 1 \\
\hline t & 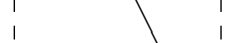 & 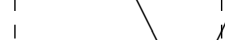 & 1 \\
\hline 1 & I & I & i \\
\hline 1 & 1 & 1 & I \\
\hline 1 & 31 & 1 & 1 \\
\hline $\begin{array}{lll}1 & 1 & 1 \\
- & - & -\end{array}$ & ' & ' & ! \\
\hline
\end{tabular}

Let $\Pi(g, P)$ denote the partition of $N$ induced by $(g, P)$. That is, $S \in \Pi(g, P)$ if and only if (i) there exists $(h, Q) \in C(g, P)$ such that $S=N(h, Q)$, or (ii) $S \in P$ such that for all $i \in S, i \notin N(g, P)$. $\Pi(\{12,23,45,56,78\},\{\{1\},\{2,3\},\{4,5\},\{6,7,8\}\})=$ $\{\{1,2,3\},\{4,5,6,7\}\}$ in the previous example.

Different coalitional networks lead to different values of overall production or overall utility to players. These various possible valuations are represented via a partition value function. A partition value function is a function $v: G^{N} \times \mathbb{P} \rightarrow \mathbb{R}$. Let $\mathcal{V}$ be the set of all possible partition value functions. A partition value function only keeps track of how the total societal value varies across different coalitional networks. The calculation of partition value is a richer object than a partition function in a partition game and/or a value function in a network game, as it allows the value generated to depend both on the coalition structure and on the network structure. A partition value function $v$ is component additive if

$$
\sum_{(h, Q) \in C(g, P)} v(h, Q)=v(g, P) .
$$

Component additivity is a condition that rules out externalities across components but still allows them within components. A coalitional network $(g, P)$ is efficient relative to a partition value function $v$ if $v(g, P) \geq v\left(g^{\prime}, P^{\prime}\right)$ for all $g^{\prime} \in G^{N}$ and all $P^{\prime} \in \mathbb{P}$.

We also wish to keep track of how that value is allocated or distributed among the players in any coalitional networks. An allocation rule is a function $Y: G^{N} \times \mathbb{P} \times \mathcal{V} \rightarrow \mathbb{R}^{N}$ such that

$$
\sum_{i \in N} Y_{i}(g, P, v)=v(g, P) \text { for all } v, g \text { and } P .
$$


It is important to note that an allocation rule depends on $g, P$ and $v$. This allows an allocation rule to take full account of a player $i$ 's role in the network and in the coalition structure. This includes not only what the network configuration and coalition structure are, but also and how the value generated depends on the overall network and coalition structure. A coalitional network $(g, P)$ is Pareto efficient relative to partition value function $v$ and allocation rule $Y$ if no $g^{\prime} \in G^{N}$ and no $P^{\prime} \in \mathbb{P}$ exist such that $Y_{i}\left(g^{\prime}, P^{\prime}, v\right) \geq Y_{i}(g, P, v)$ for all $i$ with strict inequality for some $i$.

We propose next three allocation rules that will be helpful for obtaining existence of stable coalitional networks. For any component additive partition value function $v \in \mathcal{V}$, the component-wise egalitarian allocation rule $Y^{c e}$ is such that for any $(h, Q) \in C(g, P)$ and each $i \in N(h, Q)$,

$$
Y_{i}^{c e}(g, P, v)=\frac{v(h, Q)}{\# N(h, Q)} .
$$

For any partition value function $v \in \mathcal{V}$ that is not component additive, $Y^{c e}(g, P, v)$ splits the value $v(g, P)$ equally among all players. The component-wise egalitarian rule is one in which the value of each component is split equally among the members of the component provided the partition value function is component additive.

Let $i^{S}$ be the player $i \in S, S \subset N$, such that $i \leq j$ for all $j \in S$. For any component additive partition value function $v \in \mathcal{V}$, the component-wise dictatorial allocation rule $Y^{c d}$ is such that for any $(h, Q) \in C(g, P)$ and each $S \in Q$,

$$
Y_{i}^{c d}(g, P, v)=\left\{\begin{array}{cl}
\frac{v(h, Q)}{\# Q} & i=i^{S}, \\
0 & \forall i \in S, i \neq i^{S}
\end{array}\right.
$$

For any partition value function $v \in \mathcal{V}$ that is not component additive, $Y^{c d}(g, P, v)$ splits the value $v(g, P)$ equally among all players. The component-wise dictatorial rule is one in which the value of each component is split equally among one member of each coalition belonging to the component provided this can be done.

For any component additive partition value function $v \in \mathcal{V}$, the component-wise majoritarian allocation rule $Y^{c m}$ is such that for any $(h, Q) \in C(g, P)$,

$$
Y_{i}^{c m}(g, P, v)=\left\{\begin{array}{cl}
\frac{v(h, Q)}{\sum_{S \in Q}\left[\frac{\# S}{2}+\bmod [\# S, 2]\right]} & \forall i \in S^{\prime} \subset S \\
0 & \forall i \in S^{\prime \prime} \subset S
\end{array}\right.
$$

with $S^{\prime} \cap S^{\prime \prime}=\varnothing, S^{\prime} \cup S^{\prime \prime}=S, \# S^{\prime} \geq \# S^{\prime \prime} \geq \frac{\# S}{2}-\bmod [\# S, 2]$, and $i^{S^{\prime \prime}}>j \forall j \in S^{\prime}$. For any partition value function $v \in \mathcal{V}$ that is not component additive, $Y^{c m}(g, P, v)$ splits the value $v(g, P)$ equally among all players. The component-wise majoritarian rule is one in which the value of each component is split equally among half members of each coalition belonging to the component provided this can be done. 


\section{Contractual stability}

A simple way to analyze the coalitional networks that one might expect to emerge in the long run is to examine a sort of equilibrium requirement that no coalition benefits from altering the coalitional network. What about possible deviations ? A coalitional network $\left(g^{\prime}, P^{\prime}\right)$ is obtainable from $(g, P)$ via $S, S \subset N$, if

(i) $i j \in g^{\prime}$ and $i j \notin g$ implies $\{i, j\} \subset S$, and

(ii) $i j \notin g^{\prime}$ and $i j \in g$ implies $\{i, j\} \cap S \neq \varnothing$, and

(iii) $\left\{S_{a} \backslash\left(S_{a} \cap S\right): S_{a} \in P\right\}=\left\{S_{a}^{\prime} \in P^{\prime}: S_{a}^{\prime} \subset N \backslash S\right\}$, and

(iv) $\exists\left\{S_{1}^{\prime}, S_{2}^{\prime}, \ldots, S_{m}^{\prime}\right\} \subset P^{\prime}$ such that $\cup_{a=1}^{m} S_{a}^{\prime}=S$.

Condition (i) asks that any new links that are added can only be between players inside $S$. Condition (ii) requires that there must be at least one player belonging to $S$ for the deletion of a link. ${ }^{5}$ Condition (iii) embodies the assumption that no simultaneous deviations are possible. So if players in $S$ deviate leaving their coalition in $P$, non-deviating players do not move. Condition (iv) allows deviating players in $S$ to form one or several coalitions in the new coalitional structure $P^{\prime}$. Non-deviating players do not belong to those new coalitions.

Definition 3 A coalitional network $(g, P)$ is contractually stable under the unanimity decision rule with respect to partition value function $v$ and allocation rule $Y$ if for any $S \subset N,\left(g^{\prime}, P^{\prime}\right)$ obtainable from $(g, P)$ via $S$ and $i \in S$ such that $Y_{i}\left(g^{\prime}, P^{\prime}, v\right)>Y_{i}(g, P, v)$, there exists $k \in S(j)$ with $S(j) \in P$ and $j \in S$ such that $Y_{k}\left(g^{\prime}, P^{\prime}, v\right) \leq Y_{k}(g, P, v)$.

Under the unanimity decision rule, the move from a coalitional network $(g, P)$ to any obtainable coalitional network $\left(g^{\prime}, P^{\prime}\right)$ needs the consent of every deviating player as well as the consent of every member of the initial coalitions of the deviating players. Then, a coalitional network is contractually stable ${ }^{6}$ if any deviating player or any member of

\footnotetext{
${ }^{5}$ These first two conditions have been introduced first by Jackson and van den Nouweland (2005) to define the netwoks obtainable from a given network by a coalition $S$.

${ }^{6}$ This definition of contractual stability would revert to Dutta and Mutuswami (1997) definition of strong stability if players were not able to form coalitions. The definition of strong stability of Dutta and Mutuswami considers a deviation to be valid only if all members of a deviating coalition are strictly better off, while the definition of Jackson and van den Nouweland (2005) is slightly stronger by allowing for a deviation to be valid if some members are strictly better off and others are weakly better off.
} 
the former coalitions of the deviating players is not better off from the deviation to any obtainable coalitional network $\left(g^{\prime}, P^{\prime}\right) .^{7}$

Definition 4 A coalitional network $(g, P)$ is contractually stable under the simple majority decision rule with respect to partition value function $v$ and allocation rule $Y$ if for any $S \subset N,\left(g^{\prime}, P^{\prime}\right)$ obtainable from $(g, P)$ via $S$ and $i \in S$ such that $Y_{i}\left(g^{\prime}, P^{\prime}, v\right)>Y_{i}(g, P, v)$, there exists

(i) $l \in S$ such that $Y_{l}\left(g^{\prime}, P^{\prime}, v\right) \leq Y_{l}(g, P, v)$, or

(ii) $\widehat{S} \subset S(j)$ with $S(j) \in P$ and $j \in S$ such that $Y_{k}\left(g^{\prime}, P^{\prime}, v\right) \leq Y_{k}(g, P, v)$ for all $k \in \widehat{S}$ and $\# \widehat{S} \geq \frac{1}{2} \# S(j)$.

Under the simple majority decision rule, the move from a coalitional network $(g, P)$ to any obtainable coalitional network $\left(g^{\prime}, P^{\prime}\right)$ needs the consent of every deviating player as well as the consent of more than half members of each initial coalition of the deviating players. Then, a coalitional network is contractually stable if any deviating player or half members of each former coalition of the deviating players are not better off from the deviation to any obtainable coalitional network $\left(g^{\prime}, P^{\prime}\right)$. Obviously, a coalitional network that is contractually stable under the simple majority decision rule is contractually stable under the unanimity decision rule. In fact each decision rule requires the consent of coalitional partners above some quota for a deviation not to be blocked. For instance, the simple majority decision rule reverts to a quota $q=\frac{1}{2} \# S+\bmod [\# S, 2]$ while the unanimity decision rule reverts to a quota $q=\# S .{ }^{8}$

To illustrate both the framework of coalitional networks and the concept of contractual stability we consider an alternative version of Jackson and Wolinsky (1996) symmetric connections model where players form links with each other in order to exchange information.

Example 1. The connections model with communication costs shared within groups. Players form links with each other in order to exchange information and form coalitions in order to share communication costs. If player $i$ is "connected" to player $j$, by a path of $t$ links, then player $i$ receives a payoff of $\delta^{t}$ from his indirect connection with player $j$. It

\footnotetext{
${ }^{7}$ Players cannot be farsighted in the sense that they do not forecast how others might react to their actions. Herings, Mauleon and Vannetelbosch (2004) have proposed a general concept, social rationalizability, that predicts which structures of cooperation are going to emerge among farsighted players.

${ }^{8}$ The relationship between contractual stability under any decision rule embodied by a quota is obvious: a quota $q^{\prime}<q$ refines stability. That is, the set of contractually stable coalitional networks under $q^{\prime}$ is (weakly) included in the set of contractually stable coalitional networks under $q$. Indeed, the probability to block a deviation is greater the higher the quota $q$. When the quota approaches zero $(q \rightarrow 0)$, coalitional membership has no matter in terms of consent.
} 
is assumed that $0<\delta<1$, and so the payoff $\delta^{t}$ decreases as the path connecting players $i$ and $j$ increases; thus information that travels a long distance becomes diluted and is less valuable than information obtained from a closer neighbor. Each direct link $i j$ results in a cost $c$ to both $i$ and $j$. This cost can be interpreted as the time a player must spend with another player in order to maintain a direct link. The communication costs are shared equally within groups. Player $i$ 's payoff from a network $g$ in a coalition $S(i)$ is given by

$$
Y_{i}(g, P)=\sum_{j \neq i} \delta^{t(i j)}-\frac{1}{\# S(i)} \sum_{j \in S(i)}\left(\sum_{k: j k \in g} c\right),
$$

where $t(i j)$ is the number of links in the shortest path between $i$ and $j$ (setting $t(i j)=\infty$ if there is no path between $i$ and $j$ ). Inside each group, the consent of members is needed in order to modify the network and/or the coalition structure. The contractually stable coalitional networks in case of three players under the simple majority decision rule are depicted in Figure 3.

Figure 3: Stable coalitional networks in the connections model with costs shared within groups

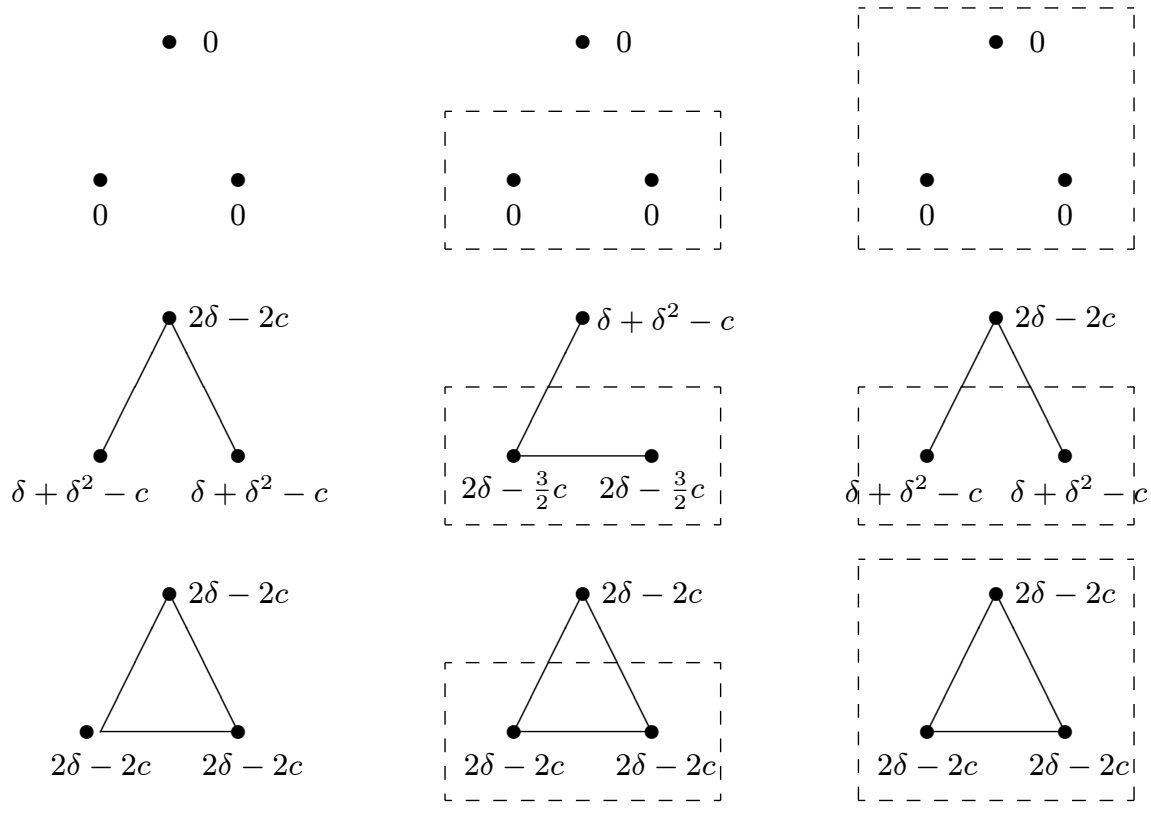

The contractually stable coalitional networks in case of three players under the simple majority decision rule are: $(\varnothing,\{\{1\},\{2\},\{3\}\})$ if and only if $c>\max \left\{\frac{3}{4}\left(\delta+\delta^{2}\right), \delta\right\}$, $(\{12,13,23\},\{\{1\},\{2\},\{3\}\})$ if and only if $c<\delta-\delta^{2},(\varnothing,\{\{1,2,3\}\})$ if and only if $c>\frac{3}{2} \delta,(\{12,13,23\},\{\{1,2,3\}\})$ if and only if $c<\min \left\{\delta-\delta^{2}, \frac{3}{4} \delta\right\},(\varnothing,\{\{i, j\},\{k\}\})$ if and only if $c>\max \left\{\frac{3}{4}\left(\delta+\delta^{2}\right), \delta\right\},(\{i j, i k\},\{\{i\},\{j\},\{k\}\})$ if and only if $\delta-\delta^{2}<c<\delta$, $(\{i j, i k\},\{\{i\},\{j, k\}\})$ if and only if $\delta-\delta^{2}<c<\delta,(\{i j, i k\},\{\{i, j\},\{k\}\})$ if and only if $c<\min \left\{\delta+\delta^{2}, \frac{4}{3} \delta\right\}$, and $(\{i j, i k, k j\},\{\{i, j\},\{k\}\})$ if and only if $c<\delta-\delta^{2}$. While the 
allocation rule depends on coalitions, the partition value function does not depend on coalitions. Hence, the efficient coalitional networks are like the efficient networks of the original symmetric connections model where each player only bears his own costs. Precisely, the efficient coalitional networks are $(\{i j, i k, k j\},\{\{i\},\{j\},\{k\}\}),(\{i j, i k, k j\},\{\{i, j\},\{k\}\})$, $(\{i j, i k, k j\},\{\{i, j, k\}\})$ if $c<\delta-\delta^{2},(\{i j, i k\},\{\{i\},\{j\},\{k\}\}),(\{i j, i k\},\{\{i, j\},\{k\}\})$, $(\{i j, i k\},\{\{i\},\{j, k\}\}),(\{i j, i k\},\{\{i, j, k\}\})$ if $\delta-\delta^{2}<c<\delta+\frac{1}{2} \delta^{2}$, and $(\varnothing,\{\{i\},\{j\},\{k\}\})$, $(\varnothing,\{\{i, j\},\{k\}\}),(\varnothing,\{\{i, j, k\}\})$ if $\delta+\frac{1}{2} \delta^{2}<c$. We have that, for any parameter values, there is always an efficient coalitional network which is contractually stable under the simple majority decision rule.

There are many contractually stable networks in the connections model when communication costs are shared within groups. However, it is easy to find an example where a contractually stable network fails to exist. For instance, take $N=\{1,2,3\}$ and let $\bar{P}=\{\{1\},\{2\},\{3\}\}$. Payoffs are given by $Y_{i}(\varnothing, \bar{P})=1, Y_{1}(\{23\}, \bar{P})=1$, $Y_{2}(\{23\}, \bar{P})=2, Y_{3}(\{23\}, \bar{P})=4, Y_{1}(\{13\}, \bar{P})=4, Y_{2}(\{13\}, \bar{P})=1, Y_{3}(\{13\}, \bar{P})=2$, $Y_{1}(\{12\}, \bar{P})=2, Y_{2}(\{12\}, \bar{P})=4, Y_{3}(\{12\}, \bar{P})=1, Y_{i}(\{13,23\}, \bar{P})=3, Y_{i}(\{12,13\}, \bar{P})=$ $3, Y_{i}(\{12,23\}, \bar{P})=3, Y_{i}(\{12,13,23\}, \bar{P})=1$, and $Y_{i}(g, P)=0 \forall i \in N, \forall P \neq \bar{P}, \forall g \in G^{N}$. The coalitional networks with positive payoffs are depicted in Figure 4.

Figure 4: Non-existence of contractually stable networks

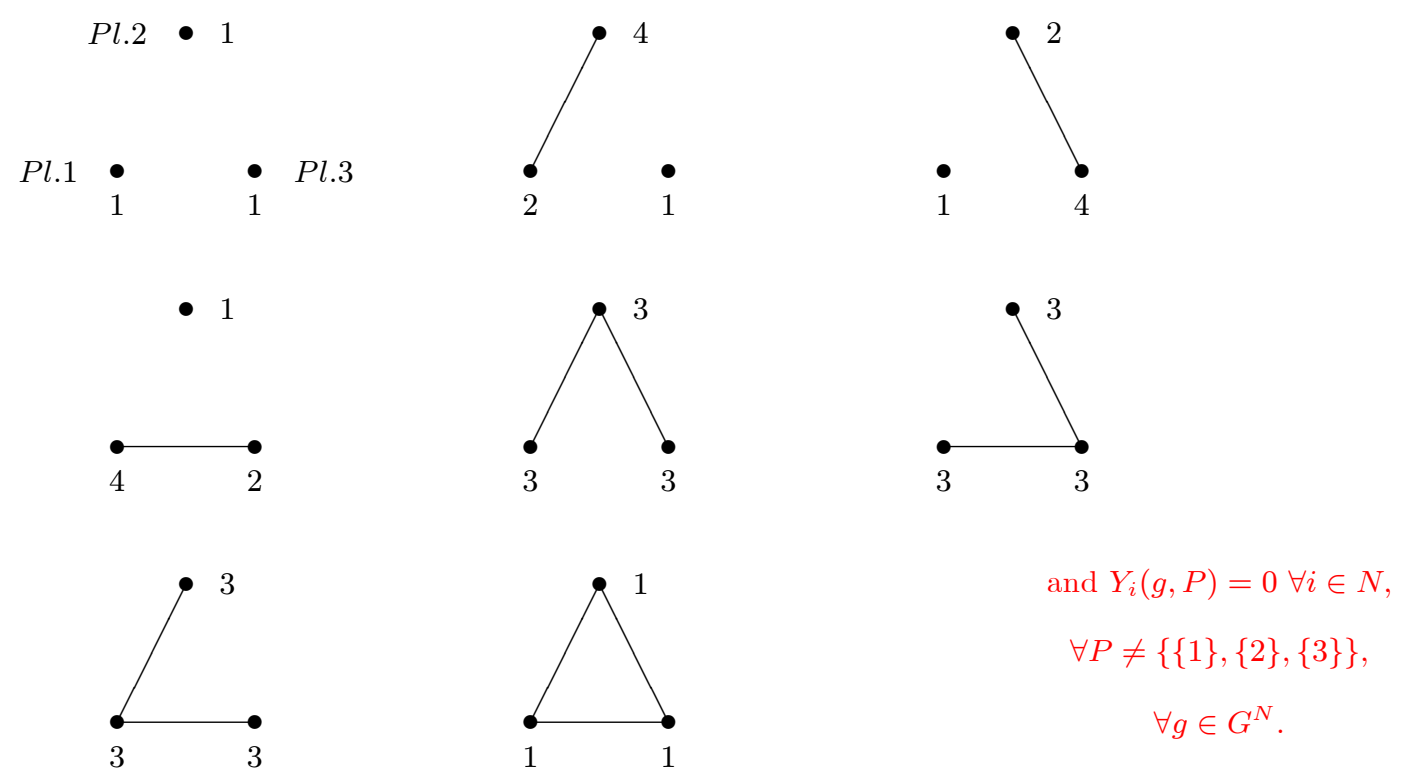




\section{$4 \quad$ Stability and efficiency}

\subsection{Allocations do not depend on coalition structures}

While contractually stable networks may fail to exist in general, we now examine the existence of contractually stable coalitional networks when values and/or allocations do not depend on coalition structures.

Proposition 1 Suppose that $v(g, P)=v(g) \forall P \in \mathbb{P}$. Under the unanimity decision rule, there always exists a contractually stable coalitional network.

Proof. Take the efficient network $g^{*}$. Then, $\left(g^{*},\{N\}\right)$ is contractually stable. Indeed, for all $\left(g^{\prime}, P\right)$ obtainable from $\left(g^{*},\{N\}\right)$ via $S$ there is a player $i \in N$ such that $Y_{i}\left(g^{\prime}, P, v\right)<Y_{i}\left(g^{*},\{N\}, v\right) .{ }^{9}$

What about the existence of contractually stable coalitional networks under the simple majority decision rule? Let $D(g, P)$ be the set of coalitional networks that are obtainable from $(g, P)$ via profitable deviations that do not modify $P$. That is, given $(g, P),\left(g^{\prime}, P\right) \in$ $D(g, P)$ if (i) $\exists S \subset N$ such that $\left(g^{\prime}, P\right)$ is obtainable from $(g, P)$ via $S$ and (ii) $Y_{i}\left(g^{\prime}, P, v\right)>$ $Y_{i}(g, P, v) \forall i \in S$. A coalitional network $(g, P)$ is critical if there exists a coalition $T$ that can block any profitable deviation obtainable from $(g, P)$ that do not modify $P$. That is, a coalitional network $(g, P)$ is critical with respect to partition value function $v$ and allocation rule $Y$ if $\exists T \subset N$ such that (i) $\# T \geq \min \{\# D(g, P), N / 2\}$ and (ii) $Y_{i}\left(g^{\prime}, P, v\right) \leq Y_{i}(g, P, v) \forall i \in T, \forall g^{\prime} \in G^{N}$ such that $\left(g^{\prime}, P\right) \in D(g, P)$.

Proposition 2 Suppose that $Y(g, P, v)=Y(g, v) \forall P \in \mathbb{P}$. If $(g,\{\{1\},\{2\}, \ldots,\{n\}\})$ is critical with respect to partition value function $v$ and allocation rule $Y$, then $\exists P \in \mathbb{P}$ such that $(g, P)$ is contractually stable under the simple majority decision rule.

Proof. Since the coalitional network $(g,\{\{1\},\{2\}, \ldots,\{n\}\})$ is critical, there exists $T \subset N$ with $\# T \geq \min \{\# D(g,\{\{1\},\{2\}, \ldots,\{n\}\}), N / 2\}$ and such that all its members do not prefer the allocation they could obtain in any coalitional network $\left(g^{\prime},\{\{1\},\{2\}, \ldots,\{n\}\}\right)$ obtainable from $(g,\{\{1\},\{2\}, \ldots,\{n\}\})$ via $S, S \cap T=\varnothing$ with $\left(g^{\prime},\{\{1\},\{2\}, \ldots,\{n\}\}\right) \in$ $D((g,\{\{1\},\{2\}, \ldots,\{n\}\}))$. Two cases have to be considered.

First, assume that $\min \{\# D(g,\{\{1\},\{2\}, \ldots,\{n\}\}), N / 2\}=\# D(g,\{\{1\},\{2\}, \ldots,\{n\}\})$. Then, there always exists a coalitional network $(g, P)$ that is contractually stable under

\footnotetext{
${ }^{9}$ In addition take any network $g$ where some player $i$ receives the maximum he can get for all $g^{\prime} \in G^{N}$. Then, $(g,\{N\})$ is contractually stable since all moves to any $\left(g^{\prime}, P\right)$ obtainable from $(g,\{N\})$ via $S$ is blocked by player $i$.
} 
the simple majority rule. We simply need to match a member of each coalition $S$ of deviators to coalitional networks $\left(g^{\prime},\{\{1\},\{2\}, \ldots,\{n\}\}\right) \in D((g,\{\{1\},\{2\}, \ldots,\{n\}\}))$ with some member of coalition $T$. Second, assume that $\min \{\# D(g,\{\{1\},\{2\}, \ldots,\{n\}\}), N / 2\}=N / 2$ which implies that $\# T \geq N / 2$. Then, the coalitional network $(g,\{N\})$ is contractually stable under the simple majority rule since the members of coalition $T$ will block any possible deviation from $(g,\{N\})$.

To illustrate this result we reconsider Jackson and Watts exchange networks model where four players get value from trading goods with each other.

Example 2. Trading networks (Jackson and Watts, 2002). Players have the same utility function, $u(x, y)=x \cdot y$. Players have a random endowment which is independently and identically distributed: $(1,0)$ with probability $\frac{1}{2}$ and $(0,1)$ with probability $\frac{1}{2}$. Players can trade with any of the other players that they are directly or indirectly linked to. For instance, if $g=\{12,34\}$ then players 1 and 2 can trade with each other; players 3 and 4 can trade with each other; but there is no trade between 12 and 34. Trade flows without friction along any "network path", and each "network component" trades to a Walrasian equilibrium. Thus, $\{12,23\}$ and $\{12,23,13\}$ lead to the same expected trades, but lead to different costs of links. What are the payoffs? Let $c=5 / 96$ be the cost of maintaining a link. Ignoring the costs of links, the expected utility of an individual is increasing and strictly concave in the number of other individuals that he is directly or indirectly linked to: (i) the utility of being alone is 0 ; (ii) the expected utility for a player of being directly or indirectly linked to one player is $1 / 8$; (iii) the expected utility for a player of being directly or indirectly linked to two players is $1 / 6$; (iv) the expected utility for a player of being directly or indirectly linked to three players is $3 / 16$. There are no pairwise nor strongly stable network in Jackson and Watts exchange networks model with four players. Suppose now that players can also form trade associations. Inside each trade association, the consent of association members is needed in order to modify the exchange network and/or the coalition structure. Under the unanimity decision rule, $(\{12,23,34\},\{N\})$ is contractually stable. Under the simple majority decision rule, $(\{12,23,34\},\{\{1,2\},\{3,4\}\})$ is contractually stable. Notice that $(\{12,23,34\},\{\{1\},\{2\},\{3\},\{4\}\})$ is critical. Indeed, the coalitions $\{1,2\}$ and $\{3,4\}$ will block all profitable deviations from the network $\{12,23,34\}$ as shown in Figure 5. 
Figure 5: A critical coalitional network in the exchange network model (all payoffs are in 96-th's)

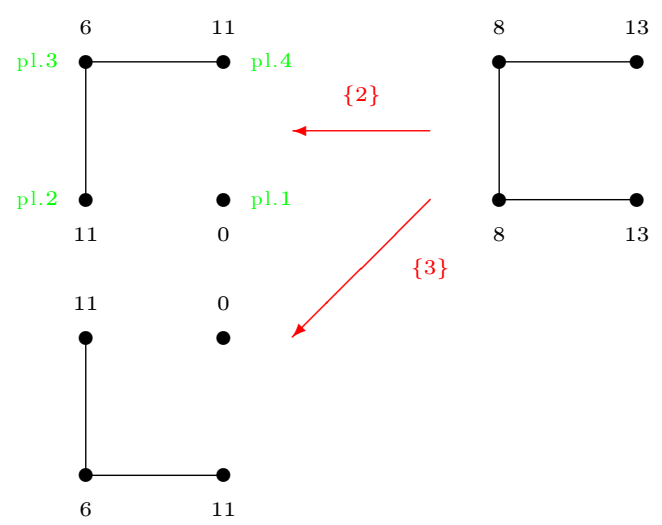

It is well-known that the relationship between stability and efficiency of networks is context dependent. Contractual stability requires the consent of group members in order to modify the network and/or the coalition structure. Does it reconcile individual or group incentives to form links and efficiency? Let us look at some classical examples to see whether requiring the consent of group members under the simple majority may help or not to reconcile stability and efficiency.

Example 3. The co-author model (Jackson and Wolinsky, 1996). Each player is a researcher who spends time writing papers and each player may belong to a research lab. If two players are linked, then they are working on a paper together. The amount of time researcher $i$ spends on a given project is inversely related to the number of projects $n_{i}$ that he is involved in. Formally, player $i$ 's payoff is given by

$$
Y_{i}(g)=\sum_{j: i j \in g}\left(\frac{1}{n_{i}}+\frac{1}{n_{j}}+\frac{1}{n_{i} n_{j}}\right)=Y_{i}(g, P) \forall P \in \mathbb{P}, n_{i}>0 .
$$

Inside each lab, the consent of lab members is needed in order to modify the network and/or the coalition structure. The possible coalitional networks and their associated payoffs in the co-author model with three players are depicted in Figure 6. 
Figure 6: Contractually stable coalitional networks in the co-author model with three players
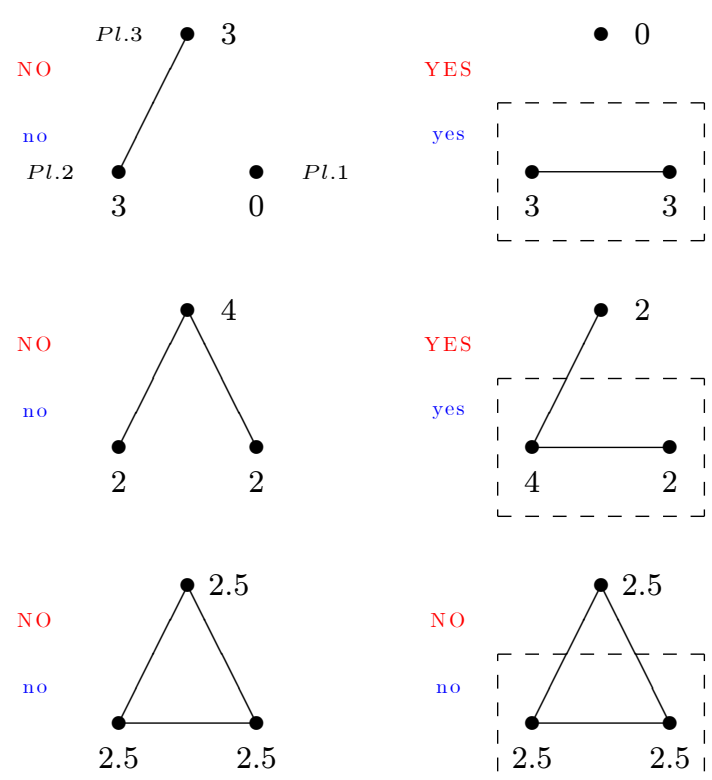
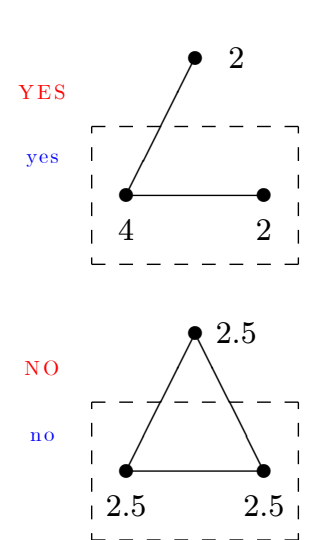
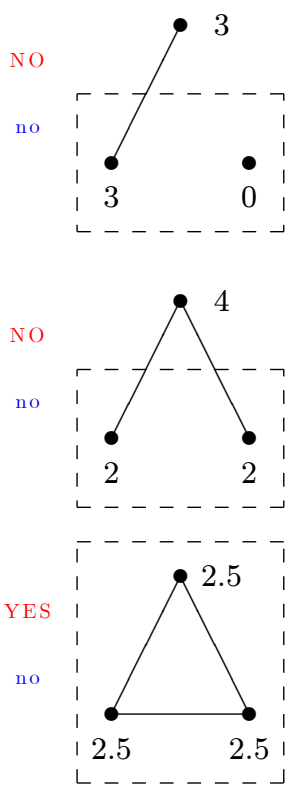
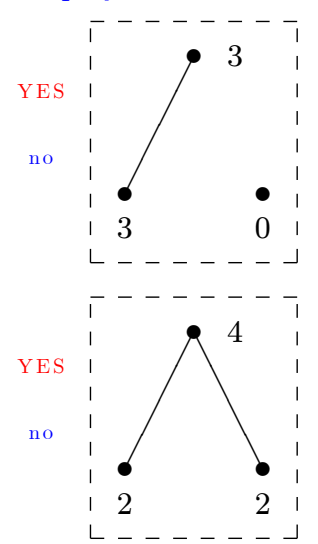

UNANIMITY RULE

simple

majority rule

In the original co-author model of Jackson and Wolinsky (1996), there does not exist strongly stable networks among three players. However, once authors may need consent of coalitional (or lab) partners, many forms of collaborations among authors become stable. Indeed, the coalitional network $(g,\{\{1\},\{2\},\{3\}\})$ is not contractually stable; but the coalitional networks $(\{12\},\{\{1,2\},\{3\}\})$ and $(\{12,23\},\{\{1,2\},\{3\}\})$ are contractually stable under the simple majority rule. For instance, $(\{12,23\},\{\{1,2\},\{3\}\})$ is contractually stable because players 1 and 3 would like to add the link 13 but this addition will be blocked by player 2 since player 1 needs the consent of player 2 (player 2 belongs to the same coalition as player 1 while player 3 is alone). Moreover, $(\{12,23\},\{\{1,2\},\{3\}\})$ is an efficient coalitional network.

Proposition 3 Take the co-author model. For n even, $(g, P)$ is contractually stable under the simple majority rule and efficient if $g$ is a network consisting of $\frac{n}{2}$ separate pairs and $P$ is a coalition structure consisting of $\frac{n}{2}$ coalitions such that $j \in S(i)$ if and only if ij $\in g$.

Proof. For $n$ even, Jackson and Wolinsky (1996, p.57) have shown that a network $g$ consisting of $\frac{n}{2}$ separate pairs is efficient. Since $v(g, P)=v(g)$ for all $P \in \mathbb{P}$, we have that $(g, P)$ is efficient for all $P \in \mathbb{P}$. Thus, for any $\left(g^{\prime}, P^{\prime}\right)$ obtainable from $(g, P)$ via deviations of $S$ we have that at least one player $j$, with $S \cap S(j) \neq \varnothing$, is worse off at $\left(g^{\prime}, P^{\prime}\right)$ compared to $(g, P)$. We show now that any of such deviations will be blocked. Two cases have to be considered. First, if $S \cap S(j) \neq \varnothing$ and $j \in S$ then $j$ will block the deviation since this deviation is making $j$ worse off. Second, if $S \cap S(j) \neq \varnothing$ and $j \notin S$ then $j$ will block 
the deviation since $j$ 's agreement is needed given that $j$ 's partner in $S(j)$ belongs to the deviating coalition $S$.

Example 4. The symmetric connections model (Jackson and Wolinsky, 1996). Each player forms links with other players in order to exchange information and each player bears his own costs of maintaining direct links. Player $i$ 's payoff from a network $g$ is given by

$$
Y_{i}(g)=\sum_{j \neq i} \delta^{t(i j)}-\sum_{j: i j \in g} c=Y_{i}(g, P) \forall P \in \mathbb{P},
$$

where $t(i j)$ is the number of links in the shortest path between $i$ and $j$ (setting $t(i j)=\infty$ if there is no path between $i$ and $j), \delta \in(0,1)$ and $c$ is the cost of maintaining a direct link. Jackson and Wolinsky have shown that for intermediate costs a conflict between stability and efficiency will arise. For $\delta<c<\delta+((N-2) / 2) \delta^{2}$, a star network encompassing all players is the unique efficient network but is not pairwise (nor strongly) stable. A star network is simply a network in which all players are linked to one central player and there are no other links. However, once we allow players to belong to coalitions and that deviations need the consent of more than half of the members of the initial coalitions of the deviating players, then the conflict between stability and efficiency may be resolved.

Proposition 4 Take the symmetric connections model. For $\delta<c<\delta+((N-2) / 2) \delta^{2}$, $(g, P)$ is contractually stable under the simple majority rule and efficient if $g$ is a star network encompassing all players and $\# S\left(i^{*}\right) \geq 2, S\left(i^{*}\right) \in P$ with $i^{*}$ being the center of the star network.

Proof. For $\delta<c<\delta+((N-2) / 2) \delta^{2}$, Jackson and Wolinsky have shown that a star network encompassing all players is efficient. Since $c>\delta$, a star network encompassing all players where $i^{*}$ is the center of the star network gives to each player $i \neq i^{*}$ his highest possible payoff he can obtain in any $g \in G^{N}$. The only player who has incentives to modify the star network encompassing all players is $i^{*}$ who obtains a negative payoff when $c>\delta$. Thus, if player $i^{*}$ is in a coalition $S\left(i^{*}\right)$ with other players (that is, $\# S\left(i^{*}\right) \geq 2$ ), those players will block any deviation from the star network encompassing all players.

Example 5. A model of buyer-seller networks (Kranton and Minehart, 2001). There is one seller who has an indivisible object for sale and $n$ potential buyers who have utilities for the object, denoted $u_{i}$, which are uniformly and independently distributed on $[0,1]$. The object to sell has no value to the seller. Each buyer knows his own valuation, but only the distribution over the buyers' valuations. The seller also knows only the distribution of buyers' valuations. The object is sold by means of a standard second-price auction. Only 
the buyers who are linked to the seller participate to the auction. Let $k$ be the number of buyers linked to the seller. For a cost per link of $c_{s}$ to the seller and $c_{b}$ to the buyer, the allocation rule for any network $g$ with $k \geq 1$ links between the buyers and the seller is

$$
Y_{i}(g)=\left\{\begin{array}{ll}
\frac{1}{k(k+1)}-c_{b} & \text { if } i \text { is a linked buyer } \\
\frac{k-1}{k+1}-k c_{s} & \text { if } i \text { is the seller } \\
0 & \text { if } i \text { is a buyer without any links. }
\end{array}=Y_{i}(g, P) \forall P \in \mathbb{P}\right.
$$

The value function is

$$
v(g)=\frac{k}{k+1}-k\left(c_{s}+c_{b}\right)=v(g, P) \forall P \in \mathbb{P},
$$

which is simply the expected value of the object to the highest valued buyer less the cost of links. Let $k^{*}$ be the number of links that maximizes $\frac{k}{k+1}-k\left(c_{s}+c_{b}\right)$.

Proposition 5 Take the buyer-seller network model. $(g, P)$ is contractually stable under the simple majority rule and efficient if $g$ is a network with $k^{*}$ links and if $P$ is a coalition structure such that $S\left(i^{*}\right) \in P$ with $i^{*}$ being the seller and buyer $j \in S\left(i^{*}\right)$ if and only if $i^{*} j \in g$.

Proof. The efficient network is one with $k^{*}$ links where

$$
k^{*}=\left\{\begin{array}{ll}
k \text { such that }(k(k+1))^{-1} \geq c_{s}+c_{b} \geq((k+1)(k+2))^{-1} & \text { if } k \leq n \\
n & \text { otherwise }
\end{array} .\right.
$$

Take $k^{*} \neq 0$. Since $\left(k^{*}\left(k^{*}+1\right)\right)^{-1}-c_{b} \geq\left(\left(k^{*}+1\right)\left(k^{*}+2\right)\right)^{-1}-c_{b}$, the buyers already linked to the seller will block the addition of new links to the efficient network. Moreover, the buyers linked to the seller have no incentives to cut their links. Since $\left(k^{*}-1\right)\left(k^{*}+1\right)^{-1}-k^{*} c_{s} \geq\left(k^{*}-2\right)\left(k^{*}\right)^{-1}-\left(k^{*}-1\right) c_{s}$ which reverts $2\left(k^{*}\left(k^{*}+1\right)\right)^{-1} \geq$ $c_{s}$, the seller does not want to cut links to the efficient network. Take now $k^{*}=0$. Since the empty network is the efficient one, if the seller wants to link to a buyer, then this buyer does not want, or vice versa.

In the original model of buyer seller networks, a conflict between stability and efficiency is likely to occur when $c_{s}>0$. However, once the seller may need the consent of the buyers linked to him, the efficient network becomes stable. While the seller and the buyers with no link have incentives to add links, the decision for adding new links will be turned down by the buyers who are already linked to the seller.

Thus, we observe that contractual stability may help to stabilize the efficient networks in some classical examples. However, contractual stability may also stabilize inefficient networks that were not stable before requiring the consent of group members. 


\subsection{Allocations depend on coalition structures}

We now study the existence of contractually stable coalitional networks when values and allocation rules depend on coalition structures. Let $g^{S}$ be the set of all subsets of $S \subset N$ of size 2. Let

$$
(h, Q)[S]=\underset{\substack{h \subset g^{S}, Q \subset P \\ \text { s.t. }(h, Q) \text { is connected }}}{\operatorname{argmax}} \frac{v(h, Q)}{\# N(h, Q)}
$$

be the connected sub-coalitional network with the highest per capita value out of those that can be formed by players in $S \subset N$. Given a component additive partition value function $v$, find a coalitional network $(g, P)^{v, c e}$ through the following algorithm. Pick some $\left(g_{1}, Q_{1}\right) \in(h, Q)[N]$. Next, pick some $\left(g_{2}, Q_{2}\right) \in(h, Q)\left[N \backslash N\left(g_{1}, Q_{1}\right)\right]$. At stage $k$ pick some $\left(g_{k}, Q_{k}\right) \in(h, Q)\left[N \backslash \cup_{i \leq k-1} N\left(g_{i}, Q_{i}\right)\right]$. Since $N$ is finite this process stops after a finite number $K$ of stages. The union of the components picked in this way defines a coalitional network $(g, P)^{v, c e}$ which is Pareto efficient. ${ }^{10}$

Proposition 6 Under a component additive partition value function $v$, a coalitional network $(g, P)^{v, c e}$ defined by the preceding algorithm is contractually stable under the simple majority decision rule and the component-wise egalitarian allocation rule $Y^{c e}$.

Proof. Given the algorithm and the component-wise egalitarian allocation rule $Y^{c e}$, the players in $N\left(g_{1}, Q_{1}\right)$ obtain the highest possible payoff they can get. So, no player in $N\left(g_{1}, Q_{1}\right)$ will deviate from $(g, P)^{v, c e}$. Players in any $N\left(g_{k}, Q_{k}\right), k=2, \ldots, K$, obtain the highest possible payoff they can get among the players in $N \backslash \cup_{i \leq k-1} N\left(g_{i}, Q_{i}\right)$. However, their payoff is smaller than the payoff of players in $N\left(g_{j}, Q_{j}\right)$ with $j=1, \ldots, k-1$. Although players in $N\left(g_{k}, Q_{k}\right)$ would like to be in any $N\left(g_{j}, Q_{j}\right)$ with $j=1, \ldots, k-1$, no player in that components would like to change its position in $N\left(g_{j}, Q_{j}\right), j=1, \ldots, k-1$, with the position of any player in $N\left(g_{k}, Q_{k}\right)$.

Let

$$
(h, Q)[S]=\underset{\substack{h \subset g^{S}, Q \subset P \text { s.t. } \\(h, Q) \text { is connected }}}{\operatorname{argmax}} \frac{v(h, Q)}{\left(\sum_{S^{\prime} \in Q}\left(\frac{\# S^{\prime}}{2}+\bmod \left[\# S^{\prime}, 2\right]\right)\right)} .
$$

be the connected sub-coalitional network out of those that can be formed by players in $S \subset N$ with the highest per capita value for a majority of players in each $S^{\prime}, S^{\prime} \in Q$. Given a component additive partition value function $v$, a similar algorithm as before provides us a coalitional network $(g, P)^{v, c m}$.

\footnotetext{
${ }^{10}$ Jackson (2005) has proposed a similar algorithm for finding a network that is pairwise stable and Pareto efficient under the classic component-wise egalitarian rule.
} 
Proposition 7 Under a component additive partition value function $v$, a coalitional network $(g, P)^{v, c m}$ defined by the preceding algorithm is contractually stable under the simple majority decision rule and the component-wise majoritarian allocation rule $Y^{\mathrm{cm}}$.

Proof. Given the algorithm and the component-wise majoritarian allocation rule $Y^{\mathrm{cm}}$, a majority of players in each coalition $S^{\prime}, S^{\prime} \in Q_{1}$, in the component $\left(g_{1}, Q_{1}\right) \in(h, Q)[N]$ obtain the highest possible payoff they can get. So, any $\left(g^{\prime}, P^{\prime}\right)$ obtainable from $(g, P)^{v, c m}$ via some coalition $S$ containing some members of $N\left(g_{1}, Q_{1}\right)$ would be blocked by the majority of players in each coalition $S^{\prime}, S^{\prime} \in Q_{1}$. Moreover, a majority of players in each coalition $S^{\prime}, S^{\prime} \in Q_{k}$, in the component $\left(g_{k}, Q_{k}\right) \in(h, Q)\left[N \backslash \cup_{i \leq k-1} N\left(g_{i}, Q_{i}\right)\right]$ obtain the highest possible payoff they can get among the players in $N \backslash \cup_{i \leq k-1} N\left(g_{i}, Q_{i}\right)$. So, any $\left(g^{\prime}, P^{\prime}\right)$ obtainable from $(g, P)^{v, c m}$ via some coalition $S \subset N \backslash \cup_{i \leq k-1} N\left(g_{i}, Q_{i}\right)$ containing some members of $N\left(g_{k}, Q_{k}\right)$ would be blocked by the majority of players in each coalition $S^{\prime}, S^{\prime} \in Q_{k}$. However, the majority of players in each $S^{\prime}, S^{\prime} \in Q_{k}$, in the component $\left(g_{k}, Q_{k}\right)$ receive a smaller payoff than a majority of players in each $S^{\prime}, S^{\prime} \in Q_{j}$, in each component $\left(g_{j}, Q_{j}\right)$, for $j=1, \ldots, k-1$. But any $\left(g^{\prime}, P^{\prime}\right)$ obtainable from $(g, P)^{v, c m}$ via $S$, involving some players in some $\left(g_{j}, Q_{j}\right), j=1, \ldots, k-1$, would be blocked by a majority of players in each coalition $S^{\prime}, S^{\prime} \in Q_{j}$.

Let

$$
(h, Q)[S]=\underset{\substack{h \subset g^{S}, Q \subset P \\ \text { s.t. }(h, Q) \text { is connected }}}{\operatorname{argmax}} \frac{v(h, Q)}{\# Q} .
$$

be the connected sub-coalitional network out of those that can be formed by players in $S \subset N$ with the highest per capita value for a single player in each $S^{\prime}, S^{\prime} \subset Q$. Given a component additive partition value function $v$, a similar algorithm as before provides us a coalitional network $(g, P)^{v, c d}$.

Proposition 8 Under a component additive partition value function $v$, a coalitional network $(g, P)^{v, c d}$ defined by the preceding algorithm is contractually stable under the unanimity decision rule and the component-wise dictatorial allocation rule $Y^{\text {cd }}$.

Proof. Given the algorithm and the component-wise dictatorial allocation rule $Y^{c d}$, a single player in each coalition $S^{\prime}, S^{\prime} \in Q_{1}$, in the component $\left(g_{1}, Q_{1}\right) \in(h, Q)[N]$ obtain the highest possible payoff he can get. So, any $\left(g^{\prime}, P^{\prime}\right)$ obtainable from $(g, P)^{v, c m}$ via some coalition $S$ containing some members of $N\left(g_{1}, Q_{1}\right)$ would be blocked by the player that obtains the highest possible payoff in each coalition $S^{\prime}, S^{\prime} \in Q_{1}$. Moreover, a single player in each coalition $S^{\prime}, S^{\prime} \in Q_{k}$, in the component $\left(g_{k}, Q_{k}\right) \in(h, Q)\left[N \backslash \cup_{i \leq k-1} N\left(g_{i}, Q_{i}\right)\right]$ 
obtain the highest possible payoff he can get among the players in $\left.N \backslash \cup_{i \leq k-1} N\left(g_{i}, Q_{i}\right)\right)$. So, any $\left(g^{\prime}, P^{\prime}\right)$ obtainable from $(g, P)^{v, c d}$ via some coalition $S \subset N \backslash \cup_{i \leq k-1} N\left(g_{i}, Q_{i}\right)$ containing some members of $N\left(g_{k}, Q_{k}\right)$ would be blocked by the player obtaining the highest payoff in each coalition $S^{\prime}, S^{\prime} \in Q_{k}$. Finally, any $\left(g^{\prime}, P^{\prime}\right)$ obtainable from $(g, P)^{v, c d}$ via $S$, involving some players in some $\left(g_{j}, Q_{j}\right), j=1, \ldots, k-1$, would be blocked by the player receiving the highest payoff in each coalition $S^{\prime}, S^{\prime} \in Q_{j}$.

Let us now reconsider the connections model with communication costs shared within groups. We have already shown that, for any parameter values, there is always an efficient coalitional network which is contractually stable in case of three players under the simple majority decision rule. But what happens for $N>3$ ? We have that, for $\delta<c<\delta+\frac{N-2}{2} \delta^{2}$, the efficient coalitional networks consist of a star network associated to any coalition structure. A coalitional network consisting of a star network associated to a coalition structure where (i) the central player is a singleton (he is alone in a coalition) is never contractually stable under the simple majority decision rule because this central player has incentives to cut links, (ii) the central player belongs to a coalition consisting of at least three players is never contractually stable under the simple majority rule because the partners of the central player have incentives to break the coalition to become singletons. The last case to be considered is the star network associated to a coalition structure where the central player forms a coalition with a single partner. If $\delta+\frac{N-2}{N} \delta<c<\delta+\frac{N-2}{2} \delta^{2}$ then both the central player and his partner have incentives to cut all their links. However, if $\delta<c<\min \left\{\delta+\frac{N-2}{N} \delta, \delta+\frac{N-2}{2} \delta^{2}\right\}$, then the central player's partner does not want to cut the link he has with the central player. We conclude that: (i) for $\delta+\frac{N-2}{N} \delta<c<\delta+\frac{N-2}{2} \delta^{2}$, no efficient coalitional network is contractually stable under the simple majority decision rule; (ii) for $\delta<c<\min \left\{\delta+\frac{N-2}{N} \delta, \delta+\frac{N-2}{2} \delta^{2}\right\}$, the coalitional network consisting of the star network associated to a coalition structure where the central player forms a coalition with a single partner is efficient and contractually stable under the simple majority decision rule. Thus, requiring the consent of group members may not be sufficient to stabilize the efficient coalitional networks. A solution to overcome the conflict between stability and efficiency in this example is to impose either the unanimity decision rule or a specific allocation rule.

\section{Discussion}

Before concluding we comment upon some of the features of the model. First, the model is general enough to study the emergence of "community structures" where links between individuals belonging to different communities are infeasible. Second, we enrich the model 
so that overlapping collections of individuals may arise.

\subsection{Community structures}

Many real world social and economic networks are composed of many communities of nodes, where the nodes of the same community are highly connected, while there are few links between the nodes of different communities. ${ }^{11}$ Suppose that two players can be linked to each other only if they belong to the same coalition. Then, the set of feasible coalitional networks becomes

$$
\left\{(g, P) \in G^{N} \times \mathbb{P}: i j \in g \text { only if } S(i)=S(j)\right\} .
$$

This situation may be interpreted as a limit case of community structures.

Proposition 9 Suppose that two players can be linked to each other only if they belong to the same coalition. Then, under a component additive partition value function $v$, efficient coalitional networks are always contractually stable under the unanimity decision rule.

If there are no externalities among coalitions (which coincide with components since players cannot be linked to players belonging to other coalitions), then it is possible to stabilize the efficient coalitional networks thanks to the unanimity decision rule, and this, whatever the allocation rule. However, once only the consent of more than half members of the initial coalitions of the deviating players is required, then we need to impose a specific allocation rule to stabilize the efficient coalitional networks.

Proposition 10 Suppose that two players can be linked to each other only if they belong to the same coalition. Under a component additive partition value function $v$, efficient community structures are contractually stable under the majority decision rule and the component-wise majoritarian allocation rule.

Proof. Let $\left\{(g, P) \in G^{N} \times \mathbb{P}: i j \in g\right.$ only if $\left.S(i)=S(j)\right\}$ be the set of feasible coalitional networks. Then, for any component additive partition value function $v$, the component-wise majoritarian allocation rule $Y^{c m}$ is such that for any $(h, S) \in C(g, P)$, $Y_{i}^{c m}(g, P, v)=v(h, S)\left[\frac{\# S}{2}+\bmod [\# S, 2]\right]^{-1} \forall i \in S^{\prime} \subset S$ and $Y_{i}^{c m}(g, P, v)=0 \forall i \in S^{\prime \prime} \subset S$, with $S^{\prime} \cap S^{\prime \prime}=\varnothing, S^{\prime} \cup S^{\prime \prime}=S, \# S^{\prime} \geq \# S^{\prime \prime} \geq \frac{\# S}{2}-\bmod [\# S, 2]$, and $i^{S^{\prime \prime}}>j \forall j \in S^{\prime}$. Let $(g, P)^{*}$ be an efficient coalitional network with $P=\left\{S_{1}^{*}, S_{2}^{*}, \ldots, S_{m}^{*}\right\}$. First, any deviation from $(g, P)^{*}$ to any $\left(g^{\prime}, P\right)$ by a coalition $S \subset S_{j}^{*}$ will be blocked because $(g, P)^{*}$ is efficient and hence in $\left(g^{\prime}, P\right)$ players in $S^{\prime} \subset S_{j}^{*}$ are worse off than in $(g, P)^{*}$ and players

\footnotetext{
${ }^{11}$ See for instance Jackson (2008) or Wasserman and Faust (1994). Research on community structures mainly deals with the detection of these communities in network data.
} 
in $S^{\prime \prime} \subset S_{j}^{*}$ are equal off. Second, any deviation from $(g, P)^{*}$ to any $\left(g^{\prime}, P^{\prime}\right)$ by a coalition $S=S_{1}^{*} \cup S_{2}^{*} \cup \ldots$ with $P^{\prime}=P \backslash\left\{S_{1}^{*}, S_{2}^{*}, \ldots\right\} \cup\left\{S_{1}^{*} \cup S_{2}^{*} \cup \ldots\right\}$ will be blocked by all the deviating players in $S_{1}^{*} \cup S_{2}^{*} \cup \ldots$ that obtain a payoff of zero. Third, any deviation from $(g, P)^{*}$ to any $\left(g^{\prime}, P^{\prime}\right)$ by a coalition $S \subset S_{j}^{*}$ with $P^{\prime}=P \backslash\left\{S_{j}^{*}\right\} \cup\left\{S_{j}^{\prime} \cup S_{j}^{\prime \prime} \cup \ldots\right\}$ and $S_{j}^{*}=S_{j}^{\prime} \cup S_{j}^{\prime \prime} \cup \ldots$ will be blocked by all the deviating players that obtain a payoff of zero in every $S_{j}^{\prime}, S_{j}^{\prime \prime}, \ldots$, with $S_{j}^{*}=S_{j}^{\prime} \cup S_{j}^{\prime \prime} \cup \ldots$ Fourth, any deviation from $(g, P)^{*}$ to any $\left(g^{\prime}, P^{\prime}\right)$ by a coalition $S$ with $P^{\prime}=P \backslash\left\{S_{1}^{*}, S_{2}^{*}\right\} \cup\{S\} \cup\left\{S_{1}^{*} \backslash\left(S_{1}^{*} \cap S\right)\right\} \cup\left\{S_{2}^{*} \backslash\left(S_{2}^{*} \cap S\right)\right\}$ will be blocked by all the deviating players that obtain a payoff of zero in $\left(g^{\prime}, P^{\prime}\right)$.

\subsection{Overlapping coalitions}

There are many situations in which players may belong simultaneously to more than one coalition. ${ }^{12}$ Overlapping groups of individuals may be involved in relationships involving reciprocity, information-sharing, working half-time in two firms, club memberships or public goods provision. A cover of $N, N=\{1, \ldots, n\}$ being the set players, is a collection of coalitions $\gamma=\left\{S_{1}, \ldots, S_{m}\right\}$ such that $\cup_{a=1}^{m} S_{a}=N$ (a coalition structure is a special case of a cover). An overlapping coalitional network $(g, \gamma)$ consists of a network $g$ and a cover $\gamma$. Let $\gamma(j)=\{S \in \gamma: j \in S\}$ be the set of coalitions in the cover $\gamma$ to which player $j$ belongs. Let $\bar{\gamma}=\{S \subset N: \# S=2\}$ be the the cover where each pair of players forms a coalition. We denote by $\Gamma$ the set of all possible covers. ${ }^{13}$ A cover value function is a function $v: G^{N} \times \Gamma \rightarrow \mathbb{R}$ which assigns a value $v(g, \gamma)$ to each overlapping coalitional network $(g, \gamma)$. An allocation rule is a function $Y: G^{N} \times \Gamma \times \mathcal{V} \rightarrow \mathbb{R}^{N}$ such that $\sum_{i \in N} Y_{i}(g, \gamma, v)=v(g, \gamma)$ for all $v, g$ and $\gamma$ which tells us how the value $v(g, \gamma)$ is distributed among the players.

An overlapping coalitional network $\left(g^{\prime}, \gamma^{\prime}\right)$ is obtainable from $(g, \gamma)$ via $S, S \subset N$, if (i) $i j \in g^{\prime}$ and $i j \notin g$ implies $\{i, j\} \subset S$, (ii) $i j \notin g^{\prime}$ and $i j \in g$ implies $\{i, j\} \cap S \neq \varnothing$, (iii) $\left\{S_{a} \backslash\left(S_{a} \cap S\right): S_{a} \in \gamma, S_{a} \notin \gamma^{\prime}\right\}=\left\{S_{a}^{\prime} \in \gamma^{\prime}: S_{a}^{\prime} \subset N \backslash S\right\}$, and (iv) $\exists\left\{S_{1}^{\prime}, S_{2}^{\prime}, \ldots, S_{m}^{\prime}\right\} \subset$ $\gamma^{\prime}$ such that $\cup_{a=1}^{m} S_{a}^{\prime}=S$. An overlapping coalitional network $(g, \gamma)$ is contractually stable under the simple majority decision rule with respect to cover value function $v$ and

\footnotetext{
${ }^{12}$ The possiblity of belonging to more than one coalition means that contracts (or coalitions) are nonexclusive.

${ }^{13}$ The notion of conference structure is any collection of conferences. The term conference refers to any set of two or more players who might meet together to discuss their cooperative plans. Thus, a conference structure is simply a cover $\gamma$ such that for any $S \in \gamma$ we have $|S| \geq 2$. The set of all possible conference structures is $\{\gamma: \forall S \in \gamma, S \subset N$ and $|S| \geq 2\}$ which is a strict subset of $\Gamma$. Myerson (1980) has studied allocations rules, which are functions mapping conference structures to payoff allocations, to describe how the outcome of a cooperative game might depend on which groups of players hold cooperative planning conferences.
} 
allocation rule $Y$ if for any $S \subset N,\left(g^{\prime}, \gamma^{\prime}\right)$ obtainable from $(g, \gamma)$ via $S$ and $i \in S$ such that $Y_{i}\left(g^{\prime}, \gamma^{\prime}, v\right)>Y_{i}(g, \gamma, v)$, there exists: (i) $l \in S$ such that $Y_{l}\left(g^{\prime}, \gamma^{\prime}, v\right) \leq Y_{l}(g, \gamma, v)$, or (ii) $\widehat{S} \subset \widehat{S}(j)$ with $\widehat{S}(j) \in \gamma(j)$ and $j \in S$ such that $Y_{k}\left(g^{\prime}, \gamma^{\prime}, v\right) \leq Y_{k}(g, \gamma, v)$ for all $k \in \widehat{S}$ and $\# \widehat{S} \geq \frac{1}{2} \# \widehat{S}(j)$. Under the simple majority decision rule, the move from an overlapping coalitional network $(g, \gamma)$ to any obtainable overlapping coalitional network $\left(g^{\prime}, \gamma^{\prime}\right)$ needs the consent of every deviating player as well as the consent of a majority of members of each coalition of the deviating players in $\gamma$.

Proposition 11 Suppose that $v(g, \gamma)=v(g) \forall \gamma \in \Gamma$. Under the simple majority decision rule, there always exists a contractually stable overlapping coalitional network.

Proof. Take the efficient network $g^{*}$. Then, $\left(g^{*}, \bar{\gamma}\right)$ is contractually stable under the simple majority rule whatever the allocation rule. Indeed, for all $\left(g^{\prime}, \gamma\right)$ obtainable from $\left(g^{*}, \bar{\gamma}\right)$ via $S$ there is a player $i \in N$ such that $Y_{i}\left(g^{\prime}, \gamma, v\right)<Y_{i}\left(g^{*}, \bar{\gamma}, v\right)$ and $\exists S^{\prime} \in \bar{\gamma}$ such that $i \in S^{\prime}$ and $S^{\prime} \cap S \neq \varnothing$.

In many situations, the cover $\bar{\gamma}$ (where each pair of players makes a binding contract) is not necessary to sustain the efficient network and a smaller number of binding contracts may be sufficient to stabilize the efficient network. It should be noted that, once forming coalitions (or making binding contracts) is costly, a conflict between stability and efficiency may again occur.

\section{Conclusion}

We have developed a theoretical framework that allows us to study which bilateral links and coalition structures are going to emerge at equilibrium. We have introduced the notion of coalitional network to represent a network and a coalition structure, where the network specifies the nature of the relationship each individual has with his coalition members and with individuals outside his coalition. To predict the coalitional networks that are going to emerge at equilibrium we have used the concept of contractual stability which requires that any change made to the coalitional network needs the consent of both the deviating players and their original coalition partners. We have shown that there always exists a contractually stable coalitional network under the simple majority decision rule and the component-wise egalitarian or majoritarian allocation rules. However, once we adopt the component-wise dictatorial allocation rule, a contractually stable coalitional network always exists only under the unanimity decision rule. Looking at some classical examples, we have shown that requiring the consent of group members under the simple majority may help to reconcile stability and efficiency. 


\section{Acknowledgments}

Ana Mauleon and Vincent Vannetelbosch are Research Associates of the National Fund for Scientific Research (FNRS), Belgium. Vincent Vannetelbosch is Associate Fellow of CEREC, Facultés Universitaires Saint-Louis. Financial support from Spanish Ministerio de Educación y Ciencia under the project SEJ2006-06309/ECON, support from the Belgian French Community's program Action de Recherches Concertée 05/10-331 (UCL), and support of a SSTC grant from the Belgian State - Belgian Science Policy under the IAP contract P6/09 are gratefully acknowledged. This paper has been presented at Concordia University, CORE, Northwestern University (GAMES 2008), SUNY at Stony Brook, Tinbergen Institute, Université Paris-Sud 11, Universidad del Pais Vasco, and Vanderbilt University. We thank the audience for helpful comments and suggestions.

\section{References}

[1] Bloch, F., "Endogenous Structures of Association in Oligopolies," Rand Journal of Economics 26, 537-556 (1995).

[2] Drèze, J.H. and J. Greenberg, "Hedonic Coalitions: Optimality and Stability," Econometrica 48, 987-1003 (1980).

[3] Dutta, B. and S. Mutuswami, "Stable Networks," Journal of Economic Theory 76, 322-344 (1997).

[4] Furusawa, T. and H. Konishi, "Free Trade Networks," Journal of International Economics 72, 310-335 (2007).

[5] Goyal, S. and S. Joshi, "Networks of Collaboration in Oligopoly," Games and Economic Behavior 43(1), 57-85 (2003).

[6] Goyal, S. and S. Joshi (2006), "Bilateralism and Free Trade," International Economic Review 47 (3), 749-778.

[7] Goyal, S. and J.L. Moraga-González, "R\&D Networks," Rand Journal of Economics 32(4), 686-707 (2001).

[8] Herings, P.J.J., A.Mauleon and V. Vannetelbosch, "Rationalizability for Social Environments," Games and Economic Behavior 49(1), 135-156 (2004).

[9] Jackson, M.O., "The Stability and Efficiency of Economic and Social Networks," in Networks and Groups: Models of Strategic Formation, edited by B. Dutta and M.O. Jackson, Springer-Verlag: Heidelberg (2003). 
[10] Jackson, M.O., "A Survey of Models of Network Formation: Stability and Efficiency," in Group Formation in Economics: Networks, Clubs and Coalitions, edited by G. Demange and M. Wooders, Cambridge University Press: Cambridge (2005).

[11] Jackson. M.O., Social and Economic Networks, Princeton University Press: Princeton (2008).

[12] Jackson, M.O. and A. van den Nouweland, "Strongly Stable Networks," Games and Economic Behavior 51, 420-444 (2005).

[13] Jackson, M.O. and A. Wolinsky, "A Strategic Model of Social and Economic Networks," Journal of Economic Theory 71, 44-74 (1996).

[14] Kranton, R. and D. Minehart, "Networks versus Vertical Integration," Rand Journal of Economics 31, 570-601 (2000).

[15] Kranton, R. and D. Minehart, "A Theory of Buyer-Seller Networks," American Economic Review 61, 485-508 (2001).

[16] Mauleon, A., J.J. Sempere-Monerris and V. Vannetelbosch, "Networks of Manufacturers and Retailers," CORE Discussion Paper 2005/41, Université catholique de Louvain, Louvain-la-Neuve, Belgium (2005).

[17] Myerson, R.B., "Conference Structures and Fair Allocation Rules," International Journal of Game Theory 9(3), 169-182 (1980).

[18] Yi, S.S., "Endogenous Formation of Customs Unions Under Imperfect Competition: Open Regionalism is Good," Journal of International Economics 41, 153-177 (1996).

[19] Wang, P. and A. Watts, "Formation of Buyer-Seller Trade Networks in a QualityDifferentiated Product Market," Canadian Journal of Economics 39, 971-1004 (2006).

[20] Wasserman, S. and K. Fraust, Social Network Analysis: Methods and Applications, Cambridge University Press: Cambridge (1994). 
Département des Sciences Économiques de l'Université catholique de Louvain

Institut de Recherches Économiques et Sociales

Place Montesquieu, 3

1348 Louvain-la-Neuve, Belgique 